\title{
BMJ Open Effectiveness of rehabilitation after a total hip arthroplasty: a protocol for an observational study for the comparison of usual care in the Netherlands versus Germany
}

\author{
Gesine H Seeber, ${ }^{1}$ Annet Wijnen, ${ }^{1,2}$ Djordje Lazovic, ${ }^{1}$ Sjoerd K Bulstra, ${ }^{2}$ \\ Günter Dietz, ${ }^{3}$ Christiaan $\mathrm{P}$ van Lingen, ${ }^{4}$ Martin Stevens ${ }^{2}$
}

To cite: Seeber GH, Wijnen A, Lazovic D, et al. Effectiveness of rehabilitation after a total hip arthroplasty: a protocol for an observational study for the comparison of usual care in the Netherlands versus Germany. BMJ Open 2017;7:e016020. doi:10.1136/ bmjopen-2017-016020

- Prepublication history for this paper is available online. To view these files please visit the journal online (http://dx.doi. org/10.1136/bmjopen-2017016020).

GHS and AW contributed equally.

Received 17 January 2017

Revised 18 May 2017

Accepted 25 May 2017

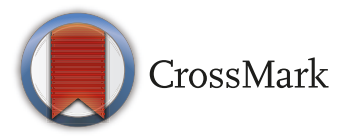

For numbered affiliations see end of article.

Correspondence to

Annet Wijnen;

annet.wijnen@uni-oldenburg.de

\section{ABSTRACT}

Introduction 0steoarthritis is the most common joint disorder worldwide. Total hip arthroplasty (THA) is considered one of the most effective treatments for endstage hip osteoarthritis. The number of THAs is expected to increase dramatically in the coming decades. Usual postoperative rehabilitation after primary THA differs between the German and the Dutch system. In the Netherlands, patients undergo fast-track surgery and are discharged into their home environment within a few days without receiving any aftercare. In Germany, patients stay in the hospital for about 12 days before being transferred to a rehabilitation centre for a period of 3 weeks. The superficially more cost-effective Dutch system of usual care after THA is judged critically in both countries due to suboptimal rehabilitation outcomes. The aim of this study is therefore to compare the Dutch with the German usual care rehabilitation after primary THA. It is hypothesised that the German procedure is more effective in terms of functional outcomes and patient satisfaction than the Dutch procedure and that in the long run the German approach is more cost-effective than the Dutch system. Methods and analysis Medical effectiveness will be assessed at four different time points by means of patient self-reported questionnaires and functional tests. Assessments include the Hip disability and Osteoarthritis Outcome Score, Patient Acceptable Symptom State, Short Form 36, EuroQol 5 Dimensions 3 Level Questionnaire, Timed Up \& Go Test and Five Times Sit-to-Stand Test. Additionally, long-term economic aspects in both countries will be assessed from a societal perspective, to get a first impression on whether cutting costs for rehabilitation, as practised in the Netherlands, really disburdens the healthcare system efficiently.

Ethics and dissemination The study is approved by the Institutional Review Boards of both University Medical Center Groningen (METc2015/483) and Hannover Medical School (no 2874-2015) and will be conducted according to the principles of the Declaration of Helsinki (64th, 2013). The results of the study will be published in international peer-reviewed scientific journals. Patient data will be presented anonymously in any publication or scientific journal.

Trial registration number DRKS00011345; Pre-results.

\section{Strength and limitations of this study}

- This is the first controlled observational trial to assess short-term as well as medium-term medical and cost effectiveness of the Dutch and/or German usual rehabilitation approach after total hip arthroplasty surgery.

- The medical evaluation includes both patient selfreported questionnaires and objective assessments. Additionally, direct and indirect costs will be evaluated from a societal perspective.

- Due to inclusion of two highly specialised hospitals and a single rehabilitation centre for German patients, the possibility of selection bias cannot be ruled out.

\section{BACKGROUND AND RATIONALE}

Osteoarthritis $(\mathrm{OA})$ is an age-related, chronic and progressive disease. $\mathrm{OA}$ is the most common joint disorder worldwide, causing pain, loss of function and disability in those affected. ${ }^{1}$ OA is recognised as a substantial source of disability and absenteeism from work with significant social and financial costs due to surgical and medical interventions and reduced work ability. ${ }^{2} \mathrm{OA}$ is most prominent in lower-extremity joints like the knee and hip. ${ }^{3}$ So far there is no cure for OA of the hip. ${ }^{45}$ Surgical treatment by means of a total hip arthroplasty (THA) is indicated for end-stage hip OA.

The prevalence of hip OA related to the total Dutch population is $\mathbf{1 5 . 9 6}$ for men and 27.03 for women, respectively, per 1000 inhabitants. ${ }^{6}$ In Germany, these numbers are roughly the same. ${ }^{7}$ The number of people with hip $\mathrm{OA}$ is expected to increase in the near future in both countries, ${ }^{6}$ due to ageing Western societies as well as growing numbers of people with overweight or obesity, which is 
considered a major risk factor for $\mathrm{OA}^{8}$ There is also an increasing trend of $\mathrm{OA}$ among the younger population. Younger patients have different demands for artificial joints as they are ordinarily more active than older patients. In Germany, 60059 patients under age 65 were provided with a primary THA in $2015 .{ }^{9}$ Within just one decade, the number of patients with hip OA in Germany aged 25-60 increased by about $25 \% .^{10}$ The prevalence of hip OA in the Dutch working-age population was 8.94 for men and 9.26 for women per 1000 inhabitants in 2011. ${ }^{6}$ As retirement age rises in Western societies, the working-age population is becoming an increasingly important group, as it is expected to return to work after surgery. It is therefore important for this group to have an optimal recovery.

At present, THA is considered one of the most successful and medically effective and cost-effective surgical treatment available for end-stage hip OA. As a result, a total of 227293 primary THAs were performed in Germany in $2015^{9}$ and 28024 in the Netherlands in $2014 .^{11}$ In the Netherlands, there is a growing trend for patients with primary THA to undergo fast-track surgery and leave the hospital within a few days. As a downside, patients are minimally supported in their rehabilitation process after hospital discharge. Moreover, postsurgical physical therapy is not regularly covered by Dutch health insurance. ${ }^{12}$ Only patients with an additional insurance coverage can claim postoperative physical therapy for a short time, depending on the insurance conditions. ${ }^{12}$ This can ultimately lead to suboptimal recovery from primary THA surgery. In Germany, patients stay in the hospital for approximately 12 days after primary THA surgery; this is followed by 3 weeks of medical rehabilitation at a rehabilitation centre to reduce OA-related symptoms such as (chronic) pain and dysfunction as well as preserve and/ or restore quality of life. For elderly patients with hip OA, this means preserving self-determined and independent living and social integration; for the younger patients, it signifies reintegration to family and daily and working life. These objectives are achieved through a multimodal, multi-professional treatment approach, including stabilisation and strengthening of joint muscles, joint protection training, provision of aids, practice of replacement functions to cope with everyday life with disabilities and nutritional counselling. ${ }^{13-15}$

Due to the expected increase in the number of patients with hip OA both in Germany and the Netherlands, the question arises of which country's postoperative policy is the most effective. As patients with OA are among the main users of the healthcare system, this increase will result in a higher socioeconomic burden of OA, especially among employable patients. ${ }^{7}$ This is why insight into the medical as well as the cost effectiveness of both postoperative policies is of the utmost relevance. The aim of this study is to compare the medical and cost effectiveness of the Dutch versus the German rehabilitation approach following primary THA.

\section{METHODS AND DESIGN}

\section{Study design}

The study will be conducted as a transnational prospective controlled observational trial in which the (cost) effectiveness of the Dutch versus the German rehabilitation approach following primary THA will be analysed. The study is a mutual project of the orthopaedic departments of University Medical Center Groningen (UMCG), the Netherlands, and Pius Hospital/Carl von Ossietzky University Oldenburg, Germany, in cooperation with affiliated hospitals. On the Dutch side, patients will undergo primary THA at Ommelander Hospital Winschoten/ Delfzijl. German patients will have surgery at the University Hospital for Orthopaedics and Trauma Surgery Pius-Hospital in Oldenburg. Inpatient rehabilitation for the German patients will take place at the rehabilitation centre Reha-Zentrum am Meer in Bad Zwischenahn.

\section{Study population}

A total of 150 (75 Dutch and 75 German) employable patients aged 18-65 who show clinical evidence of hip $\mathrm{OA}$ will be included. Clinical evidence of hip OA is based on the definition of Altman $e t a l^{16}$ : hip pain and either (A) hip internal rotation $\geq 15^{\circ}$, pain with internal rotation of the hip, morning stiffness of the hip for $\leq 60 \mathrm{~min}$ or (B) hip internal rotation $<15^{\circ}$ and hip flexion $\leq 115^{\circ}$. Those criteria have been shown to present a sensitivity of $86 \%$ and a specificity of $75 \%$. Since in Germany, patients can generally decide whether they want to do outpatient or inpatient rehabilitation after primary THA, they must additionally agree to do stationary rehabilitation at the collaborative rehabilitation centre. Patients are also required to sign a written informed consent form in order to be able to participate in the study.

Exclusion criteria are medical conditions that disallow safe participation in a rehabilitation programme, cognitive impairment and inability to sufficiently read and understand German or Dutch, as applicable.

\section{Measurements}

Measurements will be taken preoperatively $\left(\mathrm{T}_{0}\right)$ and 4 weeks $\left(T_{1}\right)$ and 12 weeks $\left(T_{2}\right)$ postoperatively. A follow-up measurement will be taken 6 months $\left(\mathrm{T}_{3}\right)$ postoperatively. Preoperative demographic data, preoperative diagnosis, height, weight, Body mass Index (BMI), American Society of Anesthesiologists classification and perioperative and postoperative complications will be recorded from electronic patient files. To assess the medical effectiveness, functional status and quality of life of patients with primary THA will be measured using patient self-reported questionnaires and objective functional measurements.

Primary outcome is the Patient Acceptable Symptom State (PASS), an instrument to measure a patient's response to a treatment or intervention. ${ }^{17}$ PASS is based on well-being or satisfaction with the actual symptoms, expressed as the score on a patient-reported outcome measure beyond which patients consider themselves well. ${ }^{18} 19$ PASS scores will be calculated from results on 
the Hip Disability and Osteoarthritis Outcome Score (HOOS). HOOS is a self-reported and disease-specific outcome measure, which consists of five subscales: pain (10 items), symptoms (5 items), activities of daily living (17 items), function in sports and recreation (4 items) and hip-related quality of life (4 items). Standardised response options are given and each question is scored from 0 to 4 on a 5-point Likert Scale. A normalised score, ranging from 0 to 100 , is subsequently calculated for each subscale, with 0 indicating extreme symptoms and 100 indicating no symptoms. The German and Dutch versions have been proven to be valid and reliable. ${ }^{2021}$ To calculate the PASS scores of HOOS, an additional question will be used asking patients about their actual satisfaction with the hip symptoms: "If you were to spend the rest of your life with the hip symptoms you have now, how would you feel?" This question will be asked in the form of a Likert Scale with four response options: very satisfied, somewhat satisfied, somewhat dissatisfied and very dissatisfied. ${ }^{18} 22$ The scores on this question are then used to calculate PASS in order to distinguish between responders and non-responders.

To measure health-related quality of life, the Short Form 36 (SF-36) and the EuroQol 5 Dimensions 3 Level Questionnaire (EQ-5D-3L) will be used. SF-36 is a widely used generic health status questionnaire. It consists of 36 questions organised into eight multi-item scales: physical functioning, role physical (limitations due to physical health problems), bodily pain, general health, vitality, social functioning, role emotional (limitations due to emotional problems) and mental health. Each raw scale score is transformed into a linear 0-100 scale. A higher score represents less disability. The German and Dutch language versions have proven to be practical, reliable and valid within a general and chronic disease population. ${ }^{2324}$ EQ-5D-3L has five dimensions: mobility, self-care, usual activities, pain/discomfort and anxiety/depression. Each dimension is divided into three degrees of severity: no problem, some problems and major problems. Actual quality of life must also be identified on the EQ-5D-3L Visual Analogue Scale. ${ }^{25}$ EQ-5D-3L has been proven to be practical, reliable and valid. ${ }^{26}$

To assess functional status objectively, the Timed Up \& Go Test (TUG) and the Five Times Sit-to-Stand Test (FTSST) will be conducted. TUG is an accepted test for measuring mobility. It is evidently reliable and valid. ${ }^{26}$ FTSST is a clinical test for assessing lower-extremity power and balance and shows good reliability. ${ }^{27}{ }^{28}$ In order to minimise learning effects as potential bias, the functional assessments will be conducted three times as per the instructions. ${ }^{29}$ In between trials, the patient rests in a sitting position for $60 \mathrm{~s}$. The mean for each test is subsequently calculated. The examiner is blinded to the results of previous measurement time points by using a blank report each time. To prevent bias, participants are not informed about their previous results.

An economic assessment comparing preoperative and 6 -month postoperative costs will also be conducted. The assessment will be done from a societal perspective, registering costs within and outside the healthcare sector. To answer the research question about cost effectiveness, direct and indirect costs will be examined. Direct costs arise from inpatient treatment, non-hospital treatment, inpatient and outpatient rehabilitation, preventive and supervisory activities, ambulance services, among others. Indirect costs arise from work absenteeism, mortality, retraining expenses, early retirement, among others. To calculate direct costs arising from hospital treatment, the Diagnosis Related Groups system will be used in both Germany and the Netherlands. ${ }^{30}$ Costs related to inpatient rehabilitation will be revealed via the set daily rate for medical rehabilitation multiplied by length of stay of an individual patient. To assess indirect costs, patients will be asked in a questionnaire to provide information about work absenteeism due to hip-related problems, necessity and frequency of visiting a medical doctor, use of pain medication, time to resumption of work, volume of employment, change of work or retraining (if any), early retirement, among others. The economic assessment will be conducted in cooperation with the medical technology assessment section of the UMCG department of epidemiology.

\section{Sample size}

The sample size calculation is based on PASS. PASS is a new approach to measure patients' response to a treatment or intervention and is therefore an easy method to establish whether a patient has achieved therapeutic success. ${ }^{17}$ According to Escobar et al, $70 \%$ of patients reached an acceptable-symptoms state at 3 months after primary THA. ${ }^{1722}$ It is hypothesised that German usual care results in a larger proportion of patients with a positive PASS at 3 months following primary THA. It is stated that a difference of $20 \%$ between the German and Dutch samples in the proportion of patients with a positive PASS is considered clinically significant. ${ }^{17} 31$ Consequently, based on the results of Escobar et al, a sample size of 60 patients in each subgroup (Germany/the Netherlands) is required to detect a $20 \%$ difference, with a power of $80 \%$ and a significance level of $0.05 .{ }^{17} 22$ Considering a drop-out rate of $20 \%$, a final enrolment of 150 patients (75 Dutch and 75 German) is needed.

\section{Statistical analysis}

Statistical analysis will be performed using IBM SPSS Statistics V.23.0 (IBM). Descriptive statistics will be used to describe the main characteristics of the two research groups as well as intraoperative and postoperative complications. For comparison between the Dutch and the German group, a multivariate analysis of variance will be done. HOOS, SF-36, EQ-5D-3L, PASS, TUG and FTSST will be included as dependent variables, and hospital and propensity score, as independent variables. Propensity scores, or the likelihood of having one of the conditions, will be estimated with the following preoperative variables: age, gender, marital status, living situation, functional 
status, comorbidity and BMI. A p-value $<0.05$ will be considered as statistically significant.

\section{ETHICS AND DISSEMINATION}

The study is approved by the Institutional Review Boards of both University Medical Groningen (METc2015/483) and Hannover Medical School (no. 2874-2015) and will be conducted according to the principles of the Declaration of Helsinki (64th, 2013). The results of the study will be published in international peer-reviewed scientific journals. Patient data will be presented anonymously in any publication or scientific journal.

\section{DISCUSSION}

The aim of this study is to compare the medical and cost effectiveness of the Dutch and German rehabilitation approaches following primary THA. Due to the increasing number of employable patients with hip OA, both Germany and the Netherlands face an enormous socioeconomic burden as patients with $\mathrm{OA}$ are among the main users of the healthcare system. To that end, insight into the medical and cost effectiveness of both postoperative policies is of the utmost relevance.

In the Netherlands, the implementation of miscellaneous evidence-based preoperative, perioperative and postoperative care components in the last decade has been demonstrated to enhance recovery using fast-track surgery. ${ }^{32} 33$ However, the quick transfer from the hospital to the home environment can lead to suboptimal rehabilitation after primary THA. Moreover, in the Dutch healthcare system, postoperative physical therapy is not regularly covered and only patients with additional insurance coverage can claim such therapy. Based on available literature, it can be stated that physical therapy should be intensive and initiated directly after THA to reduce postoperative early loss of muscle strength and function. ${ }^{32} \mathrm{~A}$ different policy is followed in Germany after discharge from the hospital, as patients follow an extensive rehabilitation programme for a 3-week period. It is hypothesised that the more comprehensive postsurgical THA rehabilitation procedure in Germany leads to better functional outcomes and more patient satisfaction than the Dutch approach. It is also hypothesised that the German procedure is more cost-effective in the long term. Should this study point out that a more intense aftercare following primary THA as handled in Germany is both medically and economically advantageous, these would be helpful arguments for Dutch surgeons to try to change their policy in cooperation with health insurance companies. For German surgeons, data of better medical outcomes compared with a non-rehabilitation approach after primary THA can be used to justify healthcare expenditures. Although unexpected, this comparison would show the Dutch system to be more cost-effective while achieving the same or even better levels of function and patient satisfaction, these would be arguments to change the German approach.

Author affiliations

${ }^{1}$ Universitätsklinik für Orthopädie und Unfallchirurgie Pius-Hospital Oldenburg, Medizinischer Campus Universität Oldenburg, Oldenburg, Germany

${ }^{2}$ Department of Orthopedics, University of Groningen, University Medical Center Groningen, Groningen, The Netherlands

${ }^{3}$ Klinik für Orthopädische und Rheumatologische Rehabilitation Reha-Zentrum am

Meer Bad Zwischenahn, Bad Zwischenahn, Germany

${ }^{4}$ Department of Orthopedics, Ommelander Ziekenhuis Groep, Winschoten, The Netherlands

Contributors GHS and MS designed the study, wrote the manuscript and arranged for funding; AW wrote the manuscript; SKB arranged for funding and designed the study; DL, GD and CPvL designed the study. All authors read and approved the final manuscript.

Funding This study is partly funded by the Medical School of Carl von Ossietzky University Oldenburg and University Medical Center Groningen. Additional funding is received from the German Statutory Pension Insurance Oldenburg/Bremen.

Competing interests None declared.

Ethics approval The study has been approved by the Institutional Review Boards of both University Medical Center Groningen (METc2015/483) and the Hannover Medical School (no 2874-2015). Signing a written informed consent form is mandatory for all eligible patients in order to be included in the study.

Provenance and peer review Not commissioned; externally peer reviewed.

Open Access This is an Open Access article distributed in accordance with the Creative Commons Attribution Non Commercial (CC BY-NC 4.0) license, which permits others to distribute, remix, adapt, build upon this work non-commercially, and license their derivative works on different terms, provided the original work is properly cited and the use is non-commercial. See: http://creativecommons.org/ licenses/by-nc/4.0/

(C) Article author(s) (or their employer(s) unless otherwise stated in the text of the article) 2017. All rights reserved. No commercial use is permitted unless otherwise expressly granted.

\section{REFERENCES}

1. Arden N, Nevitt MC. Osteoarthritis: epidemiology. Best Pract Res Clin Rheumatol 2006;20:3-25.

2. Paans N, van den Akker-Scheek I, van der Meer K, et al. The effects of exercise and weight loss in overweight patients with hip osteoarthritis: design of a prospective cohort study. BMC Musculoskelet Disord 2009;10:24.

3. Pereira D, Peleteiro B, Araújo J, et al. The effect of osteoarthritis definition on prevalence and incidence estimates: a systematic review. Osteoarthritis Cartilage 2011;19:1270-85.

4. Hochberg MC, Altman RD, Brandt KD, et al. Guidelines for the medical management of osteoarthritis. Part I. Osteoarthritis of the hip. American College of Rheumatology. Arthritis Rheum 1995;38:1535-40.

5. Peter WHF, Jansen MJ, Bloo $\mathrm{H}$, et al. Nederlands Tijdschrift voor Fysiotherapie. KNGF-richtijn arthrose heup-knie, 2010:6.

6. Poos M, Gommer AM. Neemt het aantal mensen met arthose toe of af? Volksgezondheid Toekomst Verkenning, Nationaal Kompas Volksgezondheid Bilthoven: RIVM, 2014. http://www. nationaalkompas.nl/gezondheid-en-ziekte/ziekten-en-aandoeningen/ bewegingsstelsel-en-bindweefsel/artrose/trend/. (accessed $18 \mathrm{Juli}$ 2016).

7. Rabenberg M. Arthrose. Gesundheitsberichterstattung Des. https://www.rki.de/DE/Content/Gesundheitsmonitoring/ Gesundheitsberichterstattung/GBEDownloadsT/arthrose.pdf?_blo $\mathrm{b}=$ publicationFile.

8. Jiang L, Rong J, Wang Y, et al. The relationship between body mass index and hip osteoarthritis: a systematic review and meta-analysis. Joint Bone Spine 2011;78:150-5.

9. Statistisches Bundesamt. Fallpauschalenbezogene Krankenhausstatistik (DRG-Statistik) Operationen und prozeduren der vollstationären patientinnen und patienten in Krankenhäusern - Ausführliche Darstellung: Statistisches Bundesamt. 2015 https:/ /www.destatis.de/DE/Publikationen/Thematisch/Gesundheit/Krank 
enhaeuser/OperationenProzeduren5231401157014.pdf?_blob= publicationFile (accessed 29 Nov 2016).

10. Krischak G, Kaluscha R, Kraus M, et al. Rückkehr in das Erwerbsleben nach Hüfttotalendoprothese. Unfallchirurg 2013;116:755-9.

11. LROI Raportage. Orthopedische Implantaten in Beeld: jaarrapportage uit de Landelijke Registratie Orthopedische Implantaten 2014: LROI Raportage, 2014. http://www.Iroi.nl/uploads/PR/ ev/PRevYzs9lhv9vNJo08JqwQ/LROI-jaarrapportage 2014Orthopedische-Implantaten-in-Beeld.pdf (accessed 5 Okt 2016).

12. Rijksoverheid. Zorgverzekering. https://www.rijksoverheid.nl/ onderwerpen/zorgverzekering (accessed 26 Okt 2016).

13. Heisel J, Jerosch J. Köln: Deutscher Ärzte Verlag GmbH. Rehabilitation nach Hüft- und knieendoprothese, 2007.

14. Hackenbroch MH. Arthrosen Basiswissen zu Klinik, Diagnostik und Therapie Stuttgart. Georg Thieme Verlag 2002.

15. Farin-Glattacker E, Meyer T, Vogel H. Reha-Therapiestandards Hüft- und Knie-TEP. Leitlinien für die medizinische Rehabilitation der Rentenversicherung. Deutsche Rentenversicherung Bund (Hrsg), 2016. accessed. http://www.deutsche-rentenversicherung. de/Allgemein/de/Inhalt/3_Infos_fuer_Experten/01_sozialmedizin _forschung/downloads/quali_rehatherapiestandards/TEP/rts_tep_ download.pdf? blob=publicationFile\& $v=15$.

16. Altman R, Alarcón G, Appelrouth D, et al. The American College of Rheumatology criteria for the classification and reporting of osteoarthritis of the hip. Arthritis Rheum 1991;34:505-14.

17. Rykov K, Reininga IH, Knobben BA, et al. The design of a randomised controlled trial to evaluate the (cost-) effectiveness of the posterolateral versus the direct anterior approach for THA (POLADA trial). BMC Musculoskelet Disord 2016;17:476.

18. Tubach F, Ravaud P, Martin-Mola E, et al. Minimum clinically important improvement and patient acceptable symptom state in pain and function in rheumatoid arthritis, ankylosing spondylitis, chronic back pain, hand osteoarthritis, and hip and knee osteoarthritis: results from a prospective multina. Arthritis Care Res 2012;64:1699-707.

19. Tubach F, Ravaud P, Baron G, et al. Evaluation of clinically relevant states in patient reported outcomes in knee and hip osteoarthritis: the patient acceptable symptom state. Ann Rheum Dis 2005;64:34-7.

20. de Groot IB, Reijman M, Terwee CB, et al. Validation of the Dutch version of the Hip Disability and Osteoarthritis Outcome Score. Osteoarthritis Cartilage 2009;17:132.
21. Blasimann A, Dauphinee SW, Staal JB. Translation, cross-cultural adaptation, and psychometric properties of the German version of the Hip Disability and Osteoarthritis Outcome Score. J Orthop Sports Phys Ther 2014;44:989-97.

22. Escobar A, Gonzalez M, Quintana JM, et al. Patient acceptable symptom state and OMERACT-OARSI set of responder criteria in joint replacement. Identification of cut-off values. Osteoarthritis Cartilage 2012;20:87-92.

23. Aaronson NK, Muller M, Cohen PD, et al. Translation, validation, and norming of the Dutch language version of the SF-36 Health Survey in community and chronic disease populations. J Clin Epidemiol 1998;51:1055-68.

24. Bullinger $M$. German translation and psychometric testing of the SF-36 Health Survey: preliminary results from the IQOLA Project. International Quality of Life Assessment. Soc Sci Med 1995;41:1359-66.

25. EuroQol Group. EuroQol-a new facility for the measurement of health-related quality of life. Health Policy 1990;16:199-208.

26. Oesch P, Hilfiker R, Keller S, et al. Assessments in der Rehabilitation Band 2: Bewegungsapparat. Bern: Verlag Hans Huber, 2011.

27. Lin YC, Davey RC, Cochrane T. Tests for physical function of the elderly with knee and hip osteoarthritis. Scand J Med Sci Sports 2001;11:280-6.

28. Schaubert KL, Bohannon RW. Reliability and validity of three strength measures obtained from community-dwelling elderly persons. $J$ Strength Cond Res 2005;19:717-20.

29. Yeung TS, Wessel J, Stratford PW, et al. The timed up and go test for use on an inpatient orthopaedic rehabilitation ward. J Orthop Sports Phys Ther 2008;38:410-7.

30. Busse RG, Quentin A, Wiley M. Diagnosis-Related groups in Europe. Berkshire: Open University Press, 2011.

31. Pham T, van der Heijde D, Altman RD, et al. OMERACT-OARSI initiative: Osteoarthritis Research Society International set of responder criteria for osteoarthritis clinical trials revisited. Osteoarthritis Cartilage 2004;12:389-99.

32. Bandholm T, Kehlet $H$. Physiotherapy exercise after fast-track total hip and knee arthroplasty: time for reconsideration? Arch Phys Med Rehabil 2012;93:1292-4.

33. Husted H. Fast-track hip and knee arthroplasty: clinical and organizational aspects. Acta Orthop Suppl 2012;83:1-39. 